\title{
The innate immune response to coxsackievirus B3 predicts progression to cardiovascular disease and heart failure in male mice
}

\author{
Jennifer A Onyimba ${ }^{1 \dagger}$, Michael J Coronado ${ }^{1 \dagger}$, Amanda E Garton ${ }^{1}$, Joseph B Kim', Adriana Bucek ${ }^{1}$, Djahida Bedja²,
} Kathleen L Gabrielson ${ }^{2}$, Tomas R Guilarte ${ }^{1,3}$, DeLisa Fairweather ${ }^{1,2^{*}}$

\begin{abstract}
Background: Men are at an increased risk of dying from heart failure caused by inflammatory heart diseases such as atherosclerosis, myocarditis and dilated cardiomyopathy (DCM). We previously showed that macrophages in the spleen are phenotypically distinct in male compared to female mice at $12 \mathrm{~h}$ after infection. This innate immune profile mirrors and predicts the cardiac immune response during acute myocarditis.

Methods: In order to study sex differences in the innate immune response, five male and female BALB/C mice were infected intraperitoneally with coxsackievirus B3 (CVB3) or phosphate buffered saline and their spleens were harvested $12 \mathrm{~h}$ later for microarray analysis. Gene expression was determined using an Affymetrix Mouse Gene 1.0 ST Array. Significant gene changes were verified by quantitative real-time polymerase chain reaction or ELISA.

Results: During the innate immune response to CVB3 infection, infected males had higher splenic expression of genes which are important in regulating the influx of cholesterol into macrophages, such as phospholipase $A_{2}$ $\left(\mathrm{PLA} \mathrm{A}_{2}\right)$ and the macrophage scavenger receptor compared to the infected females. We also observed a higher expression in infected males compared to infected females of squalene synthase, an enzyme used to generate cholesterol within cells, and Cyp2e1, an enzyme important in metabolizing cholesterol and steroids. Infected males also had decreased levels of the translocator protein $18 \mathrm{kDa}$ (TSPO), which binds PLA $\mathrm{A}_{2}$ and is the rate-limiting step for steroidogenesis, as well as decreased expression of the androgen receptor (AR), which indicates receptor activation. Gene differences were not due to increased viral replication, which was unaltered between sexes.

Conclusions: We found that, compared to females, male mice had a greater splenic expression of genes which are important for cholesterol metabolism and activation of the AR at $12 \mathrm{~h}$ after infection. Activation of the AR has been linked to increased cardiac hypertrophy, atherosclerosis, myocarditis/DCM and heart failure in male mice and humans.
\end{abstract}

\section{Background}

Cardiovascular disease (CVD) is the leading cause of death in the USA [1]. Heart failure can result from a number of cardiovascular conditions, including coronary artery disease, myocarditis and dilated cardiomyopathy (DCM), and men have a higher incidence and severity of these diseases than women [1-5]. Infections, such as coxsackievirus B3 (CVB3), toxins and hypersensitivity

\footnotetext{
* Correspondence: dfairwea@jhsph.edu

† Contributed equally

'Department of Environmental Health Sciences, Johns Hopkins University

Bloomberg School of Public Health, Baltimore, MD 21205, USA

Full list of author information is available at the end of the article
}

drug reactions are known to induce myocarditis $[6,7]$. Although myocarditis occurs more often in men, the rates of CVB3 infection are similar between men and women worldwide [8-10]. The factors that predict progression to myocarditis and DCM remain unknown.

We have developed a mouse model of autoimmune myocarditis and DCM using a heart-passaged strain of CVB3 that includes infectious virus and heart antigens in the inoculum [11]. All infected mouse strains develop acute myocarditis from day 8 to 12 post infection (pi), but only certain strains such as $\mathrm{A} / \mathrm{J}$ and $\mathrm{BALB} / \mathrm{c}$ develop DCM by day 35 pi [11]. Male BALB/c mice infected

\section{Biomed Central}


with heart-passaged CVB3 develop more severe acute myocarditis than females. More severe disease in males is associated with increased Toll-like receptor (TLR) 4 expression on mast cells and macrophages in the heart during acute myocarditis and in the spleen at $12 \mathrm{~h}$ pi [12-14]. TLR4 signalling following infection increases cardiac interleukin (IL)-1 $\beta$ and IL-18 resulting in a more prominent $\mathrm{T}$ helper type 1 (Th1) immune response in males $[12,13,15]$. Although males have more severe CVB3 myocarditis, the virus replicates to the same level in the hearts of both male and female mice $[12,13]$. Gonadectomy of male BALB/c mice reduces myocarditis, making males appear immunologically like females with increased IL- 4 and more alternatively activated macrophages and regulatory $\mathrm{T}$ cells in the heart [14]. Furthermore, as early as $6 \mathrm{~h}-12 \mathrm{~h}$ after infection mast cells and macrophages obtained from the spleen or peritoneum of males express more TLR4 than females, displaying the same innate immunological profile in the spleen as observed in the heart during acute myocarditis [13].

As innate immunity directs the adaptive immune response $[13,16]$, we hypothesized that sex differences in innate immune genes in the spleen would uncover pathways that are important in the susceptibility to acute myocarditis and DCM. We found that the primary gene networks elevated in males compared to females at $12 \mathrm{~h}$ after infection involved cholesterol metabolism and activation of the androgen receptor (AR) in immune cells.

\section{Methods \\ Mice}

$\mathrm{BALB} / \mathrm{cJ}(\mathrm{BALB} / \mathrm{c})$ mice were obtained from The Jackson Laboratory (ME, USA). Mice were maintained under pathogen-free conditions in the animal facility at the Johns Hopkins School of Medicine and approval was obtained from the Animal Care and Use Committee of the Johns Hopkins University for all procedures.

\section{Inoculations}

Male and female BALB/c mice (8 to 10 weeks old) were inoculated intraperitoneally (ip) with $10^{3}$ plaque forming units (PFU) of heart-passaged CVB3 (contains infectious virus and heart tissue) diluted in sterile phosphate buffered saline (PBS) and the spleen, pancreas and sera harvested at $12 \mathrm{~h}$ and $48 \mathrm{~h}$ pi. Age matched control males and females received PBS only. In separate experiments, mice received CVB3 on day 0 and echocardiography was conducted at day $10 \mathrm{pi}$ and hearts were collected for histology at day 12 and 35 pi. CVB3 (Nancy strain) was obtained from the American Type Culture Collection (ATCC, VA, USA), grown in Vero cells (ATCC) and passaged through the heart as described [11]. Mice inoculated ip with uninfected cardiac tissue supernatant diluted in PBS, or PBS alone, do not develop myocarditis [12-14]. For this reason PBS alone injections were used as uninfected controls for the innate experiments.

\section{Myocarditis and DCM}

Hearts were fixed in $10 \%$ buffered formalin, stained with haematoxylin and eosin (H\&E) and myocarditis was assessed as the percentage of the heart section with inflammation compared to the overall size of the heart section using a microscope eyepiece grid. The sections were examined by two independent investigators. The development of DCM was assessed by gross observation of histology sections at low magnification and functionally by echocardiography. Individual experiments were conducted three times with $7-10$ mice per group.

\section{Echocardiography}

Cardiac function was examined by trans-thoracic echocardiography in conscious mice using the Sequoia Acuson C256 ultrasound machine (PA, USA) equipped with a $15 \mathrm{MHz}$ linear transducer, as previously described [17]. The heart was imaged in a two-dimensional mode in the parasternal short axis view. From the M mode, the left ventricular (LV) wall thickness and chamber dimensions were determined. Ejection fraction represents stroke volume (the volume of blood ejected with each beat) divided by end diastolic LV volume.

\section{Plaque assay}

The spleen and pancreas from individual mice were homogenized at $10 \%$ weight/volume in $2 \%$ minimal essential medium (MEM) and individual supernatants were used in plaque assays to determine the level of infectious virus [11]. Virus titres are expressed as the mean plaque-forming unit (PFU)/g tissue \pm standard error of mean (SEM) and the limit of detection is $10 \mathrm{PFU} / \mathrm{g}$ of tissue. Individual experiments were conducted three times with 5 - 7 mice per group.

\section{ELISA}

Spleens were homogenized at $10 \%$ weight/volume in $2 \%$ MEM and individual supernatants used in ELISA (seven/group). Secreted PLA 2 levels were determined in homogenized supernatants using USCN Life Science kits (Wuhan, China), according to the manufacturer's instructions. PLA $\mathrm{PL}_{2}$ levels were expressed as $\mathrm{pg} / \mathrm{g}$ of spleen tissue \pm SEM. The kit did not specify sPLA ${ }_{2}$ subtype.

\section{RNA extraction and microarray}

Individual spleens from uninfected PBS-inoculated control and heart-passaged CVB3-infected male and female BALB/c mice were used for microarray analysis (five/ group, not pooled) or in a separate experiment to verify 
microarray data by real-time polymerase chain reaction (RT-PCR; seven/group, not pooled). Spleens were harvested at $12 \mathrm{~h}$ pi, flash frozen in liquid nitrogen and stored at $-80^{\circ} \mathrm{C}$. The spleens were homogenized in $2 \mathrm{~mL}$ TRIzol (Invitrogen, CA, USA) according to the manufacturer's protocol. The PureLink Micro-to-Midi Total RNA Purification System (Invitrogen) was used for extraction and purification of RNA. RNA was quantified using a NanoDrop spectrophotometer and quality assessed by RNA Nano LabChip analysis on an Agilent BioAnalyzer 2100 (Agilent Technologies, CA, USA). Processing and GeneChip analysis for microarray were performed on five samples for each treatment group. We processed $100 \mathrm{ng}$ of the total RNA for hybridization to Affymetrix Mouse Gene ST 1.0 microarrays using the Affymetrix GeneChip Whole Transcript Sense Target Labeling Assay, according to the manufacturer's protocol and previously published methods (Affymetrix, CA, USA) $[18,19]$. The Affymetrix Mouse GeneChip Gene 1.0 ST Array interrogates 28,853 well-annotated genes with 764,885 distinct probes. The expertise, facilities and instrumentation for Affymetrix GeneChip experimentation and analyses were provided and supported by the Johns Hopkins Malaria Research Institute.

Analysis of microarray data was performed with Partek Genomics Suite (GS) Version 6.4 (Partek, MO, USA). Gene expression patterns for each gene were normalized to the median array intensity for all chips and data from infected animals was normalized to uninfected PBS controls [18]. Microarray data were analysed with Partek GS software by 2-way ANOVA in order to look for significant differences between conditions (with sex and infection as factors) and then $P$ values and fold changes were generated using Fisher's least significant difference (LSD) post hoc analysis for comparisons of sex. False discovery rate (FDR) corrections for multiple comparisons (BenjaminiHochberg) were applied to reduce the total number of false-positives. Genes were considered significant if they had a $P$ value less than 0.05 . Ingenuity Pathway Analysis (IPA; Ingenuity Systems, CA, USA) was used to generate IPA network data by inputting microarray data analysed with Partek GS. Ingenuity generates networks by identifying published gene relationships and provides an IPA score ( $P$ value) indicating the likelihood that the significantly up or down-regulated genes found in our microarray would be present in a given network. The $P$ value is calculated as the $-\log$ of the Fisher's exact test.

\section{Microarray data accession number}

The Affymetrix gene expression data were deposited to the GEO http://www.ncbi.nlm.nih.gov/geo with accession number GSE26630.

\section{Validation of microarray data by quantitative RT-PCR}

Results obtained from microarray data were verified in a separate experiment using seven mice/group. Total RNA from spleens was validated by qRT-PCR using Assayon-Demand primers and probe sets and the ABI 7000 Taqman System from Applied Biosystems (CA, USA), according to Rangasamy et al. [20]. Hypoxanthine phosphoribosyltransferase 1 (HPRT) was used as a normalization control. There was no significant difference in HPRT expression in the spleen between males and females before or after infection. The mRNA data are presented as a relative gene expression (RGE). RGE is calculated as the ratio of target gene expression [fold change of messenger RNA (mRNA) of interest) to the normalization control gene expression (fold change of normalization control mRNA). Data is expressed as the mean of seven mice/group \pm SEM. Normally distributed data were analysed by the Student's $t$ test. The MannWhitney $U$ test was used to evaluate nonparametric data. A value of $P<0.05$ was considered significant.

\section{Statistical analysis}

Statistical analysis of the microarray data is described in the 'RNA Extraction and Microarray' section of the Methods. All other data were analysed by the Student's $t$ test for normally distributed data and the Mann-Whitney $U$ test for nonparametric data. A value of $P<0.05$ was considered significant.

\section{Results}

Males develop increased myocarditis and progress to heart failure and DCM

We showed previously that male BALB/c mice with acute CVB3 myocarditis develop significantly increased inflammation in the heart compared to females, while the virus replicates in the heart at the same level in both sexes $[12,13]$. Increased acute CVB3 myocarditis in males- compared to females (Figure 1A) was associated with reduced heart function with a lower ejection fraction by echocardiography at day $10 \mathrm{pi}$ (Figure 1B). An ejection fraction $\leq 45 \%$ is indicative of heart failure [21]. By day 35 pi male hearts became dilated while female hearts appeared normal (Figure 1C).

\section{Genes altered in male versus female spleens prior to and following infection}

Previously, we showed that immune factors that determine sex differences in CVB3 myocarditis, such as increased TLR4 expression on mast cells and macrophages following CVB3 infection, are upregulated as early as $12 \mathrm{~h}$ pi in the spleen of male compared to female mice $[13,22]$. As the innate immune response in the spleen is important in driving the adaptive immune response, we examined sex differences in gene 


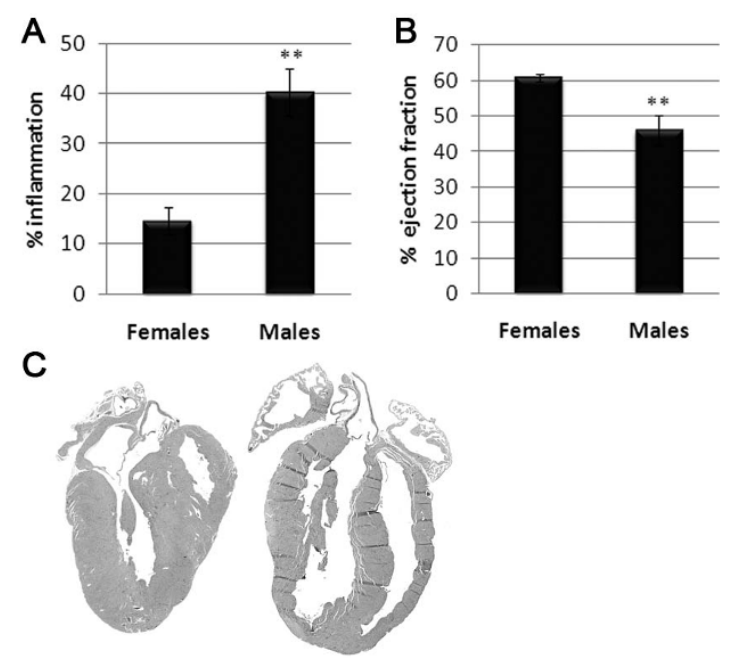

Figure 1 Males develop increased myocarditis and progress to heart failure and myocarditis and dilated cardiomyopathy (DCM). Male and female BALB/C mice were inoculated intraperitoneally with $10^{3}$ plaque forming units of heart passaged coxsackievirus B3 on day 0 and (A) myocarditis assessed histologically at day 12 post infection (pi) and (B) heart function (\% ejection fraction) assessed by echocardiography at day 10 pi. An ejection fraction $\leq 45 \%$ indicates heart failure. (C) Dilation is observed in male hearts (right) but not in female hearts (left) in histology sections at day 35 pi. Magnification $\times 5$. DCM was confirmed in males but not females at day 90 pi by echocardiography (data not shown). Data show the mean \pm standard error of the mean of $7-10$ mice per group. ${ }^{*}, P<0.01$.

expression by microarray at $12 \mathrm{~h}$ in the spleens of male and female BALB/c mice inoculated with $10^{3} \mathrm{PFU}$ CVB3 or PBS ip on day 0 . Although we conducted a two-way ANOVA analysis of data from the four groups (males versus females, PBS versus CVB3), in this paper we report only the effect of sex (we do not report gene differences between PBS versus CVB3 infected groups).

After the two-way ANOVA, we performed a post-hoc Fisher's LSD analysis in order to identify gene changes between uninfected males and uninfected females or infected males and infected females with a $P<0.05$ (Table 1). FDR corrections were applied in order to reduce the total number of false-positives. The main category of genes that was identified following FDR analysis in infected or uninfected male versus female spleens was $x$ and Y-linked genes (Table 1). These $x$ and $Y$-linked genes are routinely observed in microarray studies of sex differences in hearts from humans and mice [23].

\section{Genes increased in male versus female spleens prior to infection}

As our previous research has shown that distinct immune differences exist between infected males and infected females at $12 \mathrm{~h}$ pi in the spleen that relate to heart disease, we analysed the microarray data without correcting for multiple comparisons. The primary problem with this type of analysis is that it is likely to generate false positives. Thus, findings must be verified with other methods such as RT-PCR or ELISA. We suspect that small gene changes will be important indicators of disease susceptibility because the innate immune response to CVB3 between males and females is very similar, differing mainly in amplitude (severity) [12-14]. For example, both male and female splenocytes upregulate TLR4 following CVB3 infection but more immune cells express TLR4 in males than in females [13]. Genes found to be increased in uninfected males versus uninfected females are listed in Table 2.

Using mRNA obtained in a separate experiment from the one used for the microarray analysis, we verified by qRT-PCR that the top five genes listed in Table 2 were more highly expressed in uninfected males compared to uninfected females (Table 2, Figure 2). Several of these genes are important in regulating oxidative stress to infection including Cyp2e1, aldehyde dehydrogenase (Aldh1a7) and sulphotransferase 1e1 (Sult1e1; Figure 2). The cytochrome P450 Cyp2e1 is known to be elevated in men and is important in metabolizing cholesterol and steroids. Cyp2e1 also induces the production of reactive oxygen species (ROS) that activate proinflammatory responses and reduce viral replication [24,25]. Several other genes reported to be important in promoting heart disease and regulating lipid metabolism were elevated in the spleen of uninfected males compared to uninfected females (Table 2). These genes included: carbonic anhydrase 3 (Ca3), an enzyme known to contribute to cardiac hypertrophy and heart failure when expressed in the heart [26]; haptoglobin (Hp), a biomarker of inflammation and cardiovascular disease (CVD) [27]; and heat shock protein 90 (Hsp90aa1; Table 2). We verified that these genes had a higher expression in uninfected males by qRT-PCR even for Hsp90, which had a fold change of only 1.19 (Table 2, Figure 2). We did not verify all of the genes identified in Table 2 and so other genes in this list could include false positives. Our findings suggest that uninfected males have an underlying immune profile in the spleen that may predispose them to a proinflammatory response and inflammatory heart disease following infection.

\section{Endocrine system development and function genes increase in CVB3 infected males compared to infected females at $12 \mathrm{~h}$ pi}

Analysis of the microarray data, without correcting for multiple comparisons, revealed a number of genes expressed more highly in CVB3 infected males than in infected females (data not shown). IPA of the data found that the gene network with the most genes 
Table 1 Genes altered in the spleen of males compared to females using false discovery rate analysis

\begin{tabular}{|c|c|c|c|c|}
\hline GenBank & Gene & Gene name & Fold increase & $P$ value \\
\hline \multicolumn{5}{|c|}{ Higher expression in uninfected males } \\
\hline NM_012011 & Eif2s3y & Eukaryotic translation initiation factor 2 & 53.88 & $3.3 \times 10^{-22}$ \\
\hline NM_012008 & $D d x 3 y$ & DEAD box polypeptide, Y-linked & 38.95 & $1.8 \times 10^{-20}$ \\
\hline NM_009484 & Uty & Ubiquitously transcribed tetratricopeptide repeat gene & 23.87 & $2.3 \times 10^{-17}$ \\
\hline NM_011419 & Jarid1d & Jumonji, AT rich interactive domain & 12.21 & $3.5 \times 10^{-16}$ \\
\hline \multicolumn{5}{|c|}{ Lower expression in uninfected males } \\
\hline NR_001463 & Xist & Inactive $X$-specific transcripts & -64.65 & $4.2 \times 10^{-20}$ \\
\hline NM_009483 & Utx & Ubiquitously transcribed tetratricopeptide repeat gene & -1.45 & $1.7 \times 10^{-7}$ \\
\hline NM_012011 & Eif2s3x & Eukaryotic transl. initiation factor 2 & -1.33 & $1.2 \times 10^{-5}$ \\
\hline \multicolumn{5}{|c|}{ Higher expression in infected males } \\
\hline NM_012011 & Eif2s3y & Eukaryotic transl. initiation factor 2 & 51.01 & $4.1 \times 10^{-22}$ \\
\hline NM_012008 & Ddx3y & DEAD box polypeptide, Y-linked & 39.24 & $1.7 \times 10^{-20}$ \\
\hline NM_009484 & Uty & Ubiquitously transcribed tetratricopeptide repeat gene & 24.99 & $1.8 \times 10^{-17}$ \\
\hline NM_011419 & Jarid1d & Jumonji, AT rich interactive domain & 12.21 & $3.5 \times 10^{-16}$ \\
\hline \multicolumn{5}{|c|}{ Lower expression in infected males } \\
\hline NR_001463 & Xist & Inactive $X$-specific transcripts & -62.49 & $4.8 \times 10^{-20}$ \\
\hline NM_009483 & Utx & Ubiquitously transcribed tetratricopeptide repeat gene & -1.64 & $3.8 \times 10^{-9}$ \\
\hline NM_012010 & Eif2s3x & Eukaryotic translation initiation factor 2 & -1.33 & $1.1 \times 10^{-5}$ \\
\hline NM_011861 & Pacsin1 & Protein kinase $C$, casein kinase & -1.24 & $2.1 \times 10^{-6}$ \\
\hline
\end{tabular}

Table 2 Genes with greater expression in the spleen of males compared to females prior to infection without correction for multiple comparisons

\begin{tabular}{|c|c|c|c|c|}
\hline GenBank & Gene & Gene name & Fold increase & $P$ value \\
\hline NM_009830 & Cone2 & Cyclin E2 & 1.90 & 0.008 \\
\hline NM_021282 & Cyp2e1 & Cytochrome P450 2e1 & 1.80 & $9.4 \times 10^{-4}$ \\
\hline NM_007606 & Ca3 & Carbonic anhydrase 3 & 1.78 & 0.01 \\
\hline NM_011921 & Aldh1a7 & Aldehyde dehydrogenase family 1a7 & 1.68 & 0.03 \\
\hline NM_023135 & Sult1e1 & Sulphotransferase family 1e1 & 1.65 & 0.002 \\
\hline NM_007691 & Chek1 & Checkpoint kinase 1 & 1.64 & 0.03 \\
\hline NM_001012273 & Birc5 & Baculoviral IAP/Survivin & 1.63 & 0.04 \\
\hline NM_020504 & Cldn13 & Claudin 13 & 1.62 & 0.03 \\
\hline NM_025569 & Mgst3 & Microsomal glutathione S-transferase 3 & 1.61 & 0.04 \\
\hline NM_010360 & Gstm5 & Glutathione S-transferase, mu5 & 1.59 & 0.04 \\
\hline NM_008253 & Hmgb3 & High mobility group box 3 & 1.56 & 0.03 \\
\hline NM_017370 & $\mathrm{Hp}$ & Haptoglobin & 1.53 & 0.03 \\
\hline NM_172479 & Slc38a5 & Solute carrier family 38, member 5 & 1.53 & 0.04 \\
\hline NM_177744 & Apol10a & Apolipoprotein L 10a & 1.53 & 0.02 \\
\hline NM_028039 & Esco2 & Establishment of cohesion 1 homolog 2 & 1.53 & 0.04 \\
\hline NM_008613 & Mns1 & Meiosis-specific nuclear structural protein & 1.52 & 0.04 \\
\hline NM_009479 & Uros & Uroporphyrinogen III synthase & 1.51 & 0.03 \\
\hline NM_029522 & Gpsm2 & G-protein signalling modulator 2 & 1.50 & 0.04 \\
\hline NM_013467 & Aldh1a1 & Aldehyde dehydrogenase 1a1 & 1.49 & 0.03 \\
\hline NM_010480 & Hsp90aa1 & Heat shock protein 90kDa alpha & 1.19 & 0.04 \\
\hline
\end{tabular}




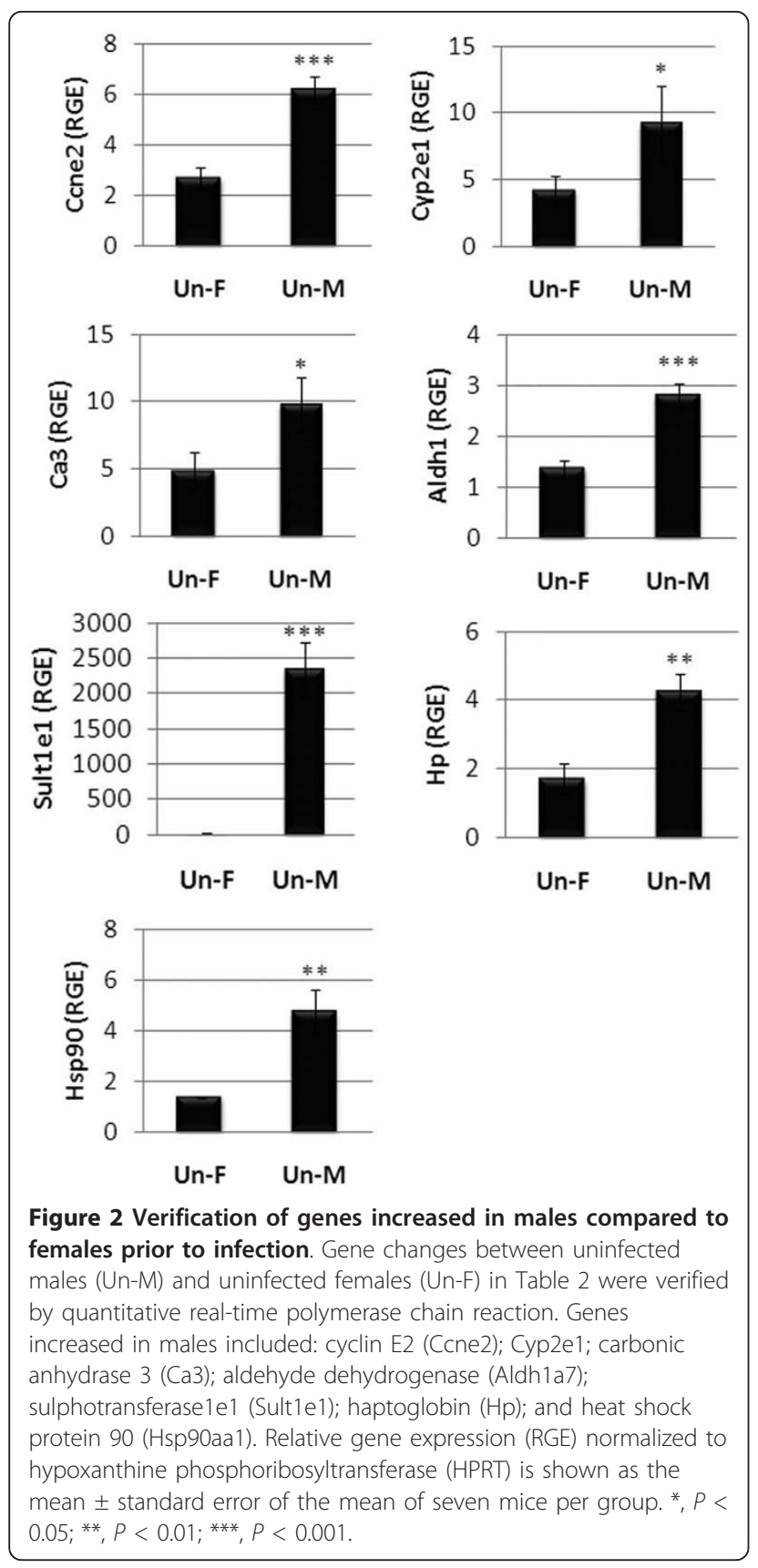

present, and thus the highest IPA score, from our study of the spleen of infected males compared to infected females involved endocrine system development and function with an IPA score of $42\left(P=1 \times 10^{-42}\right.$; Figure 3$)$. The IPA score $(P$ value) indicates the likelihood that the significantly up or down-regulated genes found in our microarray would be present in a given gene network. Using mRNA obtained in a separate experiment from the one used for the microarray analysis, we verified by qRT-PCR several of the genes shown in Figure 3. Cyp2e1 was the most highly expressed gene in this network with a fold increase of $1.8\left(P=9.4 \times 10^{-4}\right.$; Figure 3$)$. Cytochrome $\mathrm{P} 450 \mathrm{~s}$ such as aromatase play a critical role in the metabolism of steroids [28]. We were surprised to find that the AR was downregulated in the spleen of CVB3 infected males compared to infected females (Figure 3). However, it has been reported that elevated levels of testosterone decrease mRNA expression of the AR but increase AR protein and indicate activation or the use of the receptor $[28,29]$. Hsp90 acts as a chaperone preventing movement of the AR to the nucleus. We found that Hsp90 mRNA was more highly expressed in males prior to infection (Table 2, Figure 2). We verified that Cyp2e1 (Figure 3 and $4 \mathrm{~A}$ ) and the macrophage scavenger receptor (Msr1; Figures 3 and $4 \mathrm{~B}$ ), which transports oxidized lipids into macrophages, had greater expression in CVB3 infected males than infected females at $12 \mathrm{~h}$ pi. Reduced mRNA expression of the AR in CVB3 infected males compared to infected females was also verified by qRT-PCR (Figure 4C). TSPO is the rate-limiting step for steroid synthesis and is expressed in mast cells, macrophages, heart tissue and the spleen, for example [30]. We found that, similar to the AR, TSPO mRNA levels were also decreased in infected males compared to infected females at $12 \mathrm{~h}$ pi in the spleen (Figure 4D). We did not verify other genes from this network and it is likely that some of the genes shown in Figure 3 are false positives. However, we verified that Msr1, TSPO, AR and Cyp2e1, genes critical for cholesterol and steroid synthesis, are more elevated or 'activated' in the spleen of infected males compared to infected females at $12 \mathrm{~h}$ pi.

\section{CVB3 replicates at the same level in females and males} during the innate immune response

In order to examine whether sex differences in gene expression in CVB3 infected males and infected females were related to viral replication, we inoculated male and female BALB/c mice with $10^{3}$ PFU CVB3 or PBS ip on day 0 and examined the level of viral replication in the spleen and pancreas at 12 and $48 \mathrm{~h}$ pi (Figure 5). No viral replication was detected in uninfected PBS controls (data not shown) or at $12 \mathrm{~h}$ in the spleen of infected mice (Figure 5A), confirming earlier findings [22]. There was no significant difference in viral replication between CVB3 infected males and infected females at $12 \mathrm{~h}$ pi in the pancreas or at $48 \mathrm{~h}$ pi in the spleen and pancreas (the pancreas is a target organ for CVB3 infection; Figure 5A and $5 \mathrm{~B})$. Thus, altered gene expression that occurs between males and females at $12 \mathrm{~h}$ pi in the spleen by microarray is not simply due to differences in viral replication.

\section{Genes associated with cardiovascular disease increase in} CVB3 infected males compared to infected females at $12 \mathrm{~h}$ pi The network with the second highest number of genes, and thus second highest IPA score, identified by IPA of 


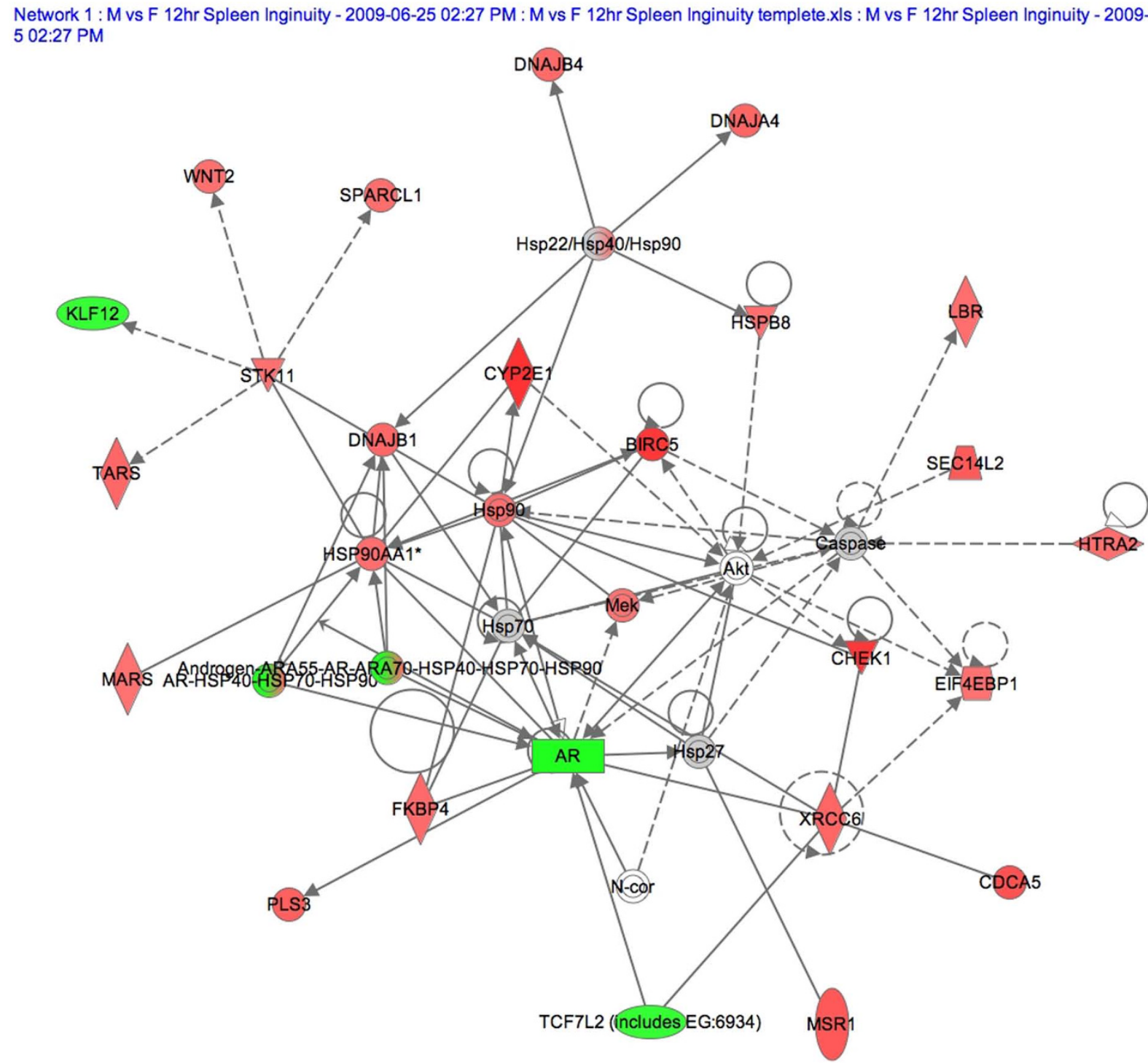

() 2000-2010 Ingenuity Systems, Inc. All rights reserved.

Figure 3 Endocrine system development and function genes increase in the spleen of CVB3 infected males at 12 h pi. Male and female BALB/C mice were inoculated intraperitoneally with $10^{3}$ plaque forming units of coxsackievirus B3 (CVB3) or phosphate buffered saline on day 0 and microarray was conducted on individual spleens at $12 \mathrm{~h}$ post infection (pi) (five/group). Ingenuity Pathway Analysis of microarray data that had not been corrected for multiple comparisons revealed that the top gene network in males involved endocrine system development and function (Network 1). Red and green represents genes significantly up- or down-regulated in CVB3 infected males compared to infected females, respectively.

data that had not been corrected for multiple comparisons in infected males compared to infected females was DNA replication, recombination and repair, with an IPA score of 41 ( $P=1 \times 10^{-41}$; data not shown). Remember, the IPA score ( $P$ value) indicates the likelihood that the significantly up or down-regulated genes found in our microarray would be present in a given gene network. This gene network also received the highest IPA score for data that had not been corrected for multiple comparisons between uninfected males and uninfected females, with an IPA score of $46\left(P=1 \times 10^{-46}\right.$; data not shown). None of the other networks comparing uninfected males to uninfected females corresponded to those revealed for CVB3 infected males versus infected females, which indicates that infection activated a distinct immune profile in the spleen of males that was not simply due to baseline sex differences. 

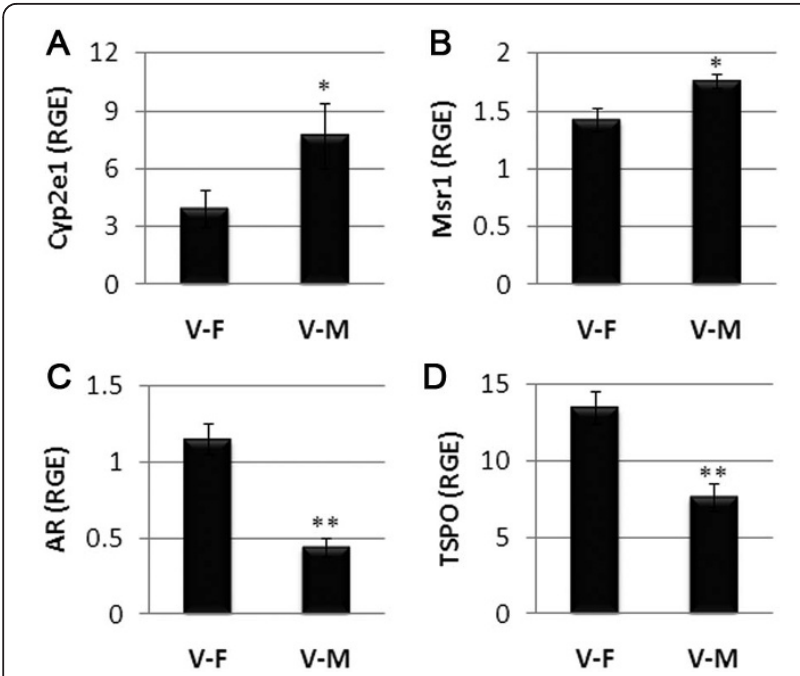

Figure 4 Verification of endocrine system development and function genes. Using a separate experiment from the one used for the microarray, gene changes in Figure 3 were verified by quantitative real-time polymerase chain reaction between coxsackievirus B3 infected males (V-M) and infected females ( $\mathrm{V}-\mathrm{F})$. Verified genes included: (A) Cyp2e1; (B) the macrophage scavenger receptor (Msr1); (C) the androgen receptor (AR); and (D) translocator protein $18 \mathrm{kDa}$ (TSPO). Relative gene expression (RGE) normalized to hypoxanthine phosphoribosyltransferase (HPRT) is shown as the mean \pm standard error of the mean of seven mice per group. ${ }^{*}, P<0.05 ;{ }^{* *}, P<0.01$

Unexpectedly, we found that the networks with the 3rd and 5th highest IPA scores in the spleen of CVB3 infected males compared to those in the infected females from data that had not been corrected for multiple comparisons included cardiovascular system development and function with an IPA score of $38(P=1 \times$ $10^{-38}$; data not shown) and cardiovascular and metabolic disease with an IPA score of $29\left(P=1 \times 10^{-29}\right.$; Figure 6, Table 3). These CVD gene networks were not observed in uninfected males versus uninfected females. Genes with well known roles in promoting CVD that were more highly expressed in the spleen of CVB3 infected males compared to infected females at $12 \mathrm{~h}$ pi included carbonic anhydrase (Ca1-Ca3; Figure 6, Table 2 and 3) and $\mathrm{PLA}_{2}$ (Cpla2, Pla2g4c, Pla2g12a; Figure 6, Table 3) [26]. Elevated lipoprotein-associated (Lp)-PLA 2 is associated with the development of atherosclerosis and stroke [31]. Other key proteins in this network included: farnesyl-diphosphate faresyltranserferase 1 (Fdft1), also called squalene synthase, which increases intracellular cholesterol levels [32]; MAP2 kinase 3 (Map2k3) which has been shown to contribute to sex-based differences in myocardial remodelling and heart failure [33,34]; and the chemokine CXCL12 which regulates neutrophil and macrophage function (Figure 6, Table 3) [35].

Using separate experiments from the one used for the microarray, we verified by qRT-PCR or ELISA several of

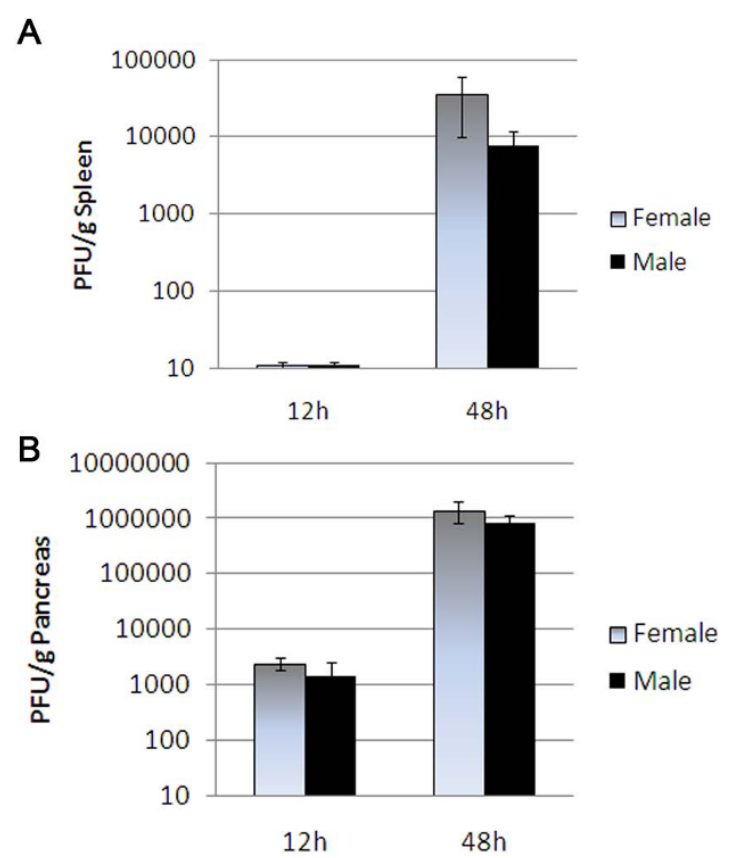

Figure 5 CVB3 replicates at the same level in males and females during the innate immune response. Male and female $\mathrm{BALB} / \mathrm{C}$ mice were inoculated intraperitoneally with $10^{3}$ plaque forming units (PFU) of coxsackievirus B3 (CVB3)or phosphate buffered saline (PBS) on day 0 and the level of viral replication in the spleen (A) and pancreas (B) examined at $12 \mathrm{~h}$ and $48 \mathrm{~h}$ post infection. No viral replication was detected in uninfected PBS controls. No significant differences in viral replication were observed in CVB3 infected males compared to infected females. Data show the mean \pm standard error of the mean of 5 - 7 mice pergroup.

the genes shown in Table 3 . We confirmed that $\mathrm{PLA}_{2}$ was increased in the spleen of CVB3 infected males versus infected females using ELISA (Figure 7A) and that infected males had greater mRNA expression of carbonic anhydrase 1 ( $\mathrm{Ca} 1$; Figure $7 \mathrm{~B}$ ) and squalene synthase (Fdft1; Figure 7C) by qRT-PCR. Although we were able to confirm that carbonic anhydrase $3(\mathrm{Ca} 3)$ expression was greater in uninfected males than uninfected females (Figure 2), we were unable to confirm that $\mathrm{Ca} 3$ was elevated in CVB3 infected males by RT-PCR. We did not verify other genes in Table 3 and so the list could include other false positives. However, we were able to verify that $\mathrm{Ca} 1, \mathrm{PLA}_{2}$ and squalene synthase had higher expression in the spleen of CVB3 infected males than in infected females at $12 \mathrm{~h}$ pi (Figure 7). Thus, a number of genes associated with the development of CVD and heart failure, and genes that regulate cholesterol metabolism, show greater expression in the spleen of CVB3 infected males during innate immunity. Our findings indicate that these genes may be involved in initiating the immune response that results in inflammatory heart disease. Further studies are necessary to confirm their role. 

Network 5 : M vs F $12 \mathrm{hr}$ Spleen Inginuity - 2009-06-25 02:27 PM : M vs F 12hr Spleen Inginuity templete.xls : M vs F 12hr Spleen Inginuit
y-2009-06-25 02:27 PM

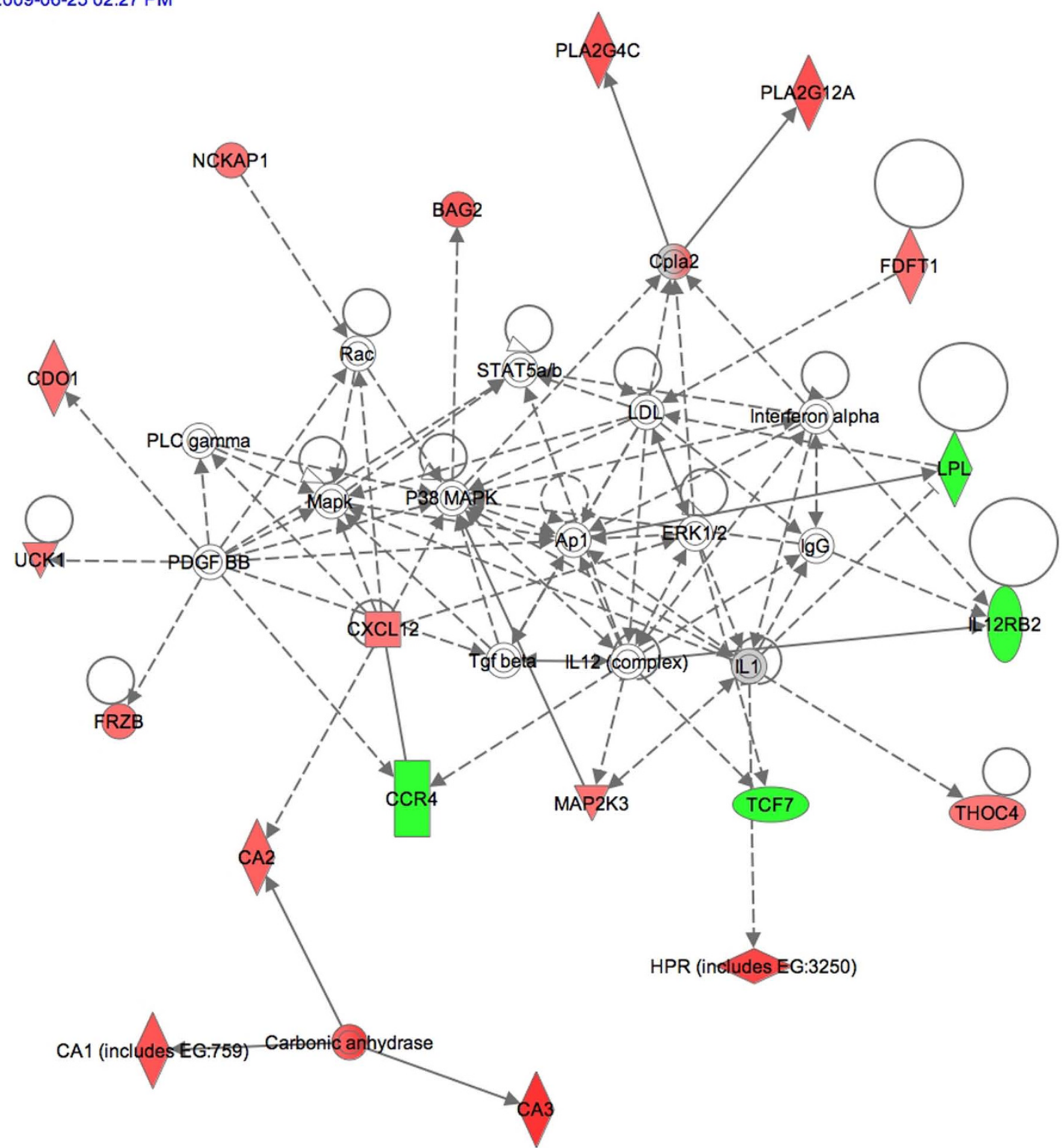

(9) 2000-2010 Ingenuity Systems, Inc. All rights reserved.

Figure 6 Cardiovascular disease genes increase in the spleen of CVB3 infected males at $\mathbf{1 2} \mathbf{h}$ pi. Male and female BALB/C mice were inoculated intraperitoneally with $10^{3}$ plaque forming units of coxsackievirus B3 (CVB3) or phosphate buffered saline on day 0 and a microarray conducted on individual spleens at $12 \mathrm{~h}$ pi (five/group). Ingenuity Pathway Analysis of microarray data that had not been corrected for multiple comparisons revealed that genes associated with cardiovascular disease were increased in CVB3 infected males compared to infected females (Network 5). Red and green represents genes significantly up- or down-regulated, respectively.

\section{Discussion}

Recent evidence indicates that the innate immune response is critical in determining the adaptive response to infection or vaccination. We previously showed that as early as $12 \mathrm{~h}$ pi males respond to CVB3 infection with an elevated proinflammatory response [13]. Elevated TLR4 expression on mast cells and macrophages in the spleen and heart of CVB3 infected males not only increases acute myocarditis but induces expression of the profibrotic cytokine IL- $1 \beta$ resulting in cardiac dilatation and heart failure later [13-15,36]. In this study, we examined the sex differences in gene expression in the spleen of 
Table 3 Cardiovascular disease, metabolic disease, and genetic disorder genes (Network 5)

\begin{tabular}{|c|c|c|c|c|}
\hline GenBank & Gene & Gene name & Fold increase & $P$ value \\
\hline \multicolumn{5}{|c|}{ Higher expression in males } \\
\hline NM_007606 & Ca3 & Carbonic anhydrase 3 & 1.78 & 0.01 \\
\hline NM_183423 & Pla2g12a & Phospholipase A2, group XIIA & 1.43 & 0.02 \\
\hline NM_001004762 & Pla2g4c & Phospholipase A2, group IVC & 1.40 & 0.02 \\
\hline NM_145392 & Bag2 & BCL2-associated athanogene & 1.32 & 0.02 \\
\hline NM_009801 & $\mathrm{Ca} 2$ & Carbonic anhydrase 2 & 1.31 & 0.03 \\
\hline NM_011356 & Frzb & Frizzled-related protein & 1.20 & 0.02 \\
\hline NM_033037 & Cdo1 & Cysteine deoxygenase 1 & 1.19 & 0.01 \\
\hline NM_010191 & Fdft1 & Farnesyl-diphosphate farnesyltransferase 1 & 1.18 & 0.02 \\
\hline NM_008928 & Map2k3 & MAP2 kinase 3 & 1.15 & 0.03 \\
\hline NM_011568 & Thoc4 & THO complex 4 & 1.13 & 0.04 \\
\hline NM_011675 & Uck1 & Uridine-cytidine kinase 1 & 1.13 & 0.04 \\
\hline NM_001012477 & $\mathrm{CxCl} 12$ & Chemokine ligand 12 & 1.12 & 0.005 \\
\hline NM_016965 & Nckap1 & NCK-associated protein 1 & 1.12 & 0.04 \\
\hline \multicolumn{5}{|c|}{ Lower expression in males } \\
\hline NM_009331 & Tcf7 & Transcr. factor $7, T$ cell-specific & -1.33 & 0.02 \\
\hline NM_009916 & Ccr4 & Chemokine receptor 4 & -1.31 & 0.0002 \\
\hline NM_008509 & Lpl & Lipoprotein lipase & -1.31 & 0.005 \\
\hline NM_008354 & $1112 \mathrm{rb} 2$ & Interleukin 12 receptor, beta 2 & -1.28 & 0.01 \\
\hline
\end{tabular}

$\mathrm{BALB} / \mathrm{c}$ mice prior to, and during, the innate immune response to CVB3 infection. We found that FDR analysis of sex differences following infection revealed mainly differences in $x$ and Y-linked genes. However, by analysing the microarray data without correcting for multiple comparisons we were able to identify by Partek and Ingenuity software and to verify by qRT-PCR and ELISA a number of other gene changes between sexes. Similar to our findings, several studies in rodents and humans examining sex differences in heart disease using FDR analysis yielded few gene changes aside from $\times$ and $\mathrm{Y}$-linked genes $[4,23,34,37]$. This is an important issue for the study of sex differences in heart disease and other chronic inflammatory conditions. These findings suggest that small gene changes are likely to be important in driving sex differences in inflammatory diseases. Most of the low-fold gene changes we verified from the IPA networks involved genes critical to the regulation of cholesterol and steroid synthesis in immune cells and in macrophages in particular (Figure 8).

For many years it has been known that underlying sex differences exist in the expression of hepatic enzymes such as cytochrome P450 s, sulphotransferases and glutathione $S$-transferases $[24,38]$. We verified that male spleens have a higher expression of Cyp2e1 and Sult1e1 (Table 2, Figures 2 and 4). These enzymes are important in metabolizing drugs, steroids, fatty acids and environmental chemicals, as well as amplifying a protective immune response against infection. Thus, elevated levels of these enzymes in the spleen of males may predispose them to increased inflammation following CVB3 infection.

In addition, we found that males respond to CVB3 infection by upregulating genes specifically involved in cholesterol metabolism (Figure 8). Squalene synthase (Fdft1) regulates intracellular cholesterol levels particularly the endogenous production of steroid hormones, vitamin D, bile acids and lipoprotein particles (Figures 6, 7 and 8, Table 3). Squalene synthase inhibitors have been found to reduce plasma levels of total- and low-density lipoprotein (LDL)-cholesterol in clinical studies [32]. Another source of cholesterol for macrophages comes from extracellular LDL-cholesterol. LDL is recruited from the circulation to the cell wall where it becomes oxidized by secreted PLA $\left(\mathrm{SPLA}_{2}\right)$ among other factors [39]. The superfamily of $\mathrm{PLA}_{2}$ enzymes includes 15 distinct groups that fall into four main categories: secreted $\left(\mathrm{sPLA}_{2}\right)$; cytosolic $\left(\mathrm{cPLA}_{2}\right)$; calcium-independent PLA 2 ; and Lp-PLA 2 [40]. Approximately $80 \%$ of $\mathrm{Lp}-\mathrm{PLA}_{2}$ is associated with LDL in the sera, and elevated levels of Lp-PLA 2 and $\mathrm{SPLA}_{2} \mathrm{~S}$ occur in patients with atherosclerosis $[31,40,41]$. PLA 2 catalyzes the removal of fatty acids from the $s n-2$ position of membrane phospholipids releasing arachidonic acid for the generation of lipid mediators that are crucial for many inflammatory processes such as leukotrienes, prostaglandins and thromboxanes $[42,43]$. Increased sPLA 2 levels have been associated with a number of inflammatory conditions including: rheumatoid arthritis; sepsis; psoriasis; pancreatitis; and cancer $[43,44]$. cPLA 2 levels increase in phagocytic 


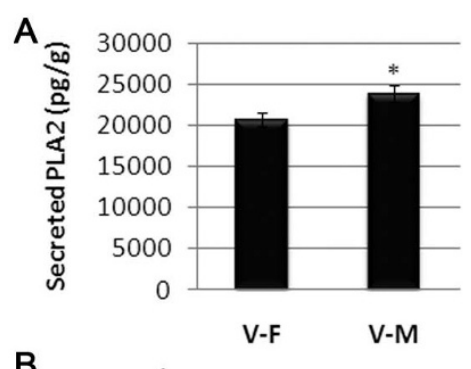

B

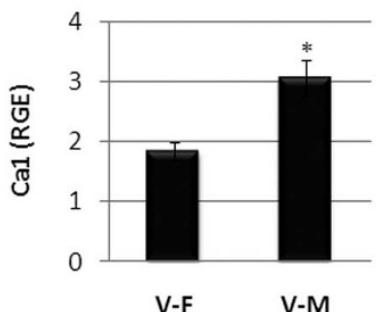

C

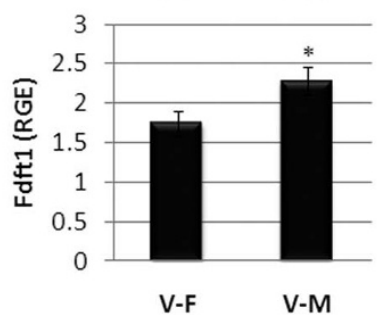

Figure 7 Verification of cardiovascular disease genes in infected males. Using separate experiments from the one used for the microarray, gene changes in Table 3 were verified by ELISA or quantitative real-time polymerase chain reaction (qRT-PCR) between coxsackievirus B3 - infected males ( $V-M)$ and infected females (V-F). Secreted phospolipase $A_{2}$ (PLA2) was verified in the spleen at (A) 48 h post infection (pi) by ELISA, while (B) carbonic anhydrase 1 (Ca1) and $(C)$ squalene synthase (Fdft1) were verified by qRT-PCR at $12 \mathrm{~h}$ pi. Relative gene expression (RGE) normalized to hypoxanthine phosphoribosyltransferase is shown as the mean \pm standard error of the mean of seven mice per group. ${ }^{*}, P<0.05$.

cells, such as mast cells, macrophages and neutrophils, following their activation where cPLA 2 has been found to be essential for phagocytic function [42]. Similarly, $\mathrm{SPLA}_{2}$ is secreted from mast cells and macrophages following activation. In this study we were able to verify increased sPLA2 in the spleen of CVB3 infected males compared to infected females (Figures 6 and 7A, Table 3). PLA 2 is also a ligand for TSPO and so elevated levels of $\mathrm{PLA}_{2}$ in the spleen of males could facilitate AR-mediated activation of immune cells during the innate immune response to CVB3 infection (Figure 8). Oxidized-LDL becomes a ligand for LDL receptors (LDLR) and macrophage scavenger receptors (Msr1) that transport cholesterol into macrophages to be metabolized to steroids and other lipid mediators that increase proinflammatory responses (Figure 8) [39]. Our observation of greater expression of $\mathrm{sPLA}_{2}$ and Msr1 in the spleen of CVB3 infected males may allow oxidation and uptake of LDL-cholesterol and activation of

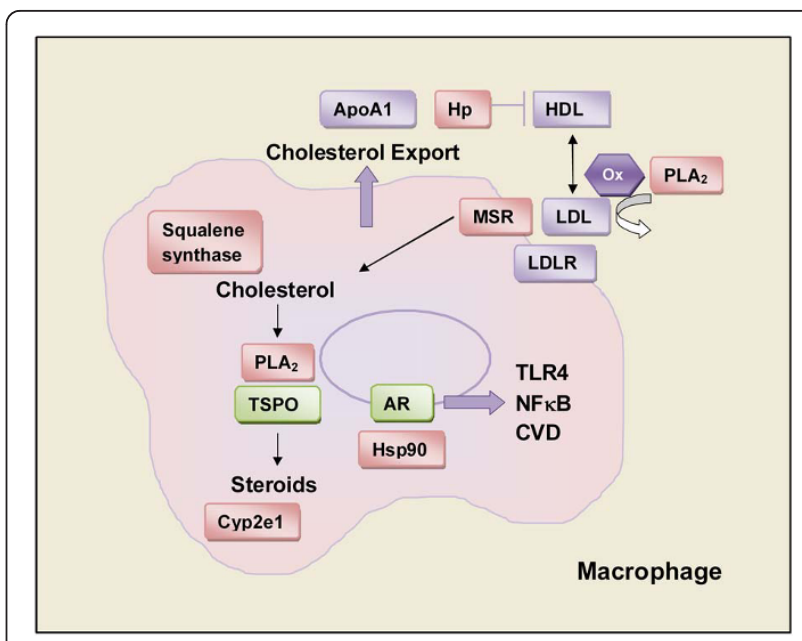

Figure 8 Genes increased in males involve regulation of cholesterol metabolism and steroidogenesis. In this study we verified that a number of genes that are important in regulating cholesterol influx into cells and steroidogenesis are more highly expressed in the spleen of males prior to, or during, the innate immune response to coxsackievirus B3 (CVB3) infection. Genes with a higher expression in males are shown in pink boxes, genes with lower expression in males in green boxes and genes we did not examine in purple boxes. We suggest the following hypothesis as one possible scenario leading to increased inflammation in males. Haptoglobin ( $\mathrm{Hp}$ ) is known to bind Apo-A1 preventing the conversion of proinflammatory low-density lipoproteins (LDLs) to anti-inflammatory high-density lipoproteins (HDLs), thereby increasing the $L D L$ levels. Elevated phospholipase $A_{2}\left(P L A_{2}\right)$ in males may facilitate the oxidation (Ox) of LDL allowing it to bind the macrophage scavenger receptor (Msr1/MSR) and import LDLcholesterol into immune cells, particularly macrophages. Increased levels of squalene synthase in males may facilitate intracellular cholesterol synthesis in immune cells. PLA 2 is an endogenous ligand for translocator protein $18 \mathrm{kDa}$ (TSPO), the rate-limiting step for steroidogenesis within cells. Cyp2e1 participates in the metabolism of cholesterol and steroids and contributes to an increased proinflammatory response in males. Increased production of androgens within macrophages allows the androgen receptor (AR) to release from its chaperone Hsp90 and move to the nucleus to stimulate the proinflammatory immune response associated with increased myocarditis and heart failure in males following CVB3 infection.

TSPO for the production of steroids in splenic macrophages (Figures 4 and 7). Interestingly, PLA 2 expression in the heart was found to be a sex-related gene that predicted the progression to heart failure in CVD patients [34]. Haptoglobin was also more highly expressed in the spleen of males prior to infection (Table 2, Figure 2). By binding Apo-AI, haptoglobin inhibits the transfer of lipids from pro-inflammatory LDL particles to anti-inflammatory HDL thereby potentially increasing LDL levels (Figure 8) $[39,45]$.

Findings from our study that examined sex differences in the spleen correspond closely to clinical studies of sex differences in gene expression in CVD and heart failure patients. Sex studies of normal and diseased 
hearts in rodents and humans have found sex differences in carbonic anhydrase (Table 2 and 3 ), $\mathrm{PLA}_{2}$ (Table 3), Map2k3 (Table 3) and the AR (Figure 3 and 4 ; see, for example, $[4,23,34,37,46]$. With an ever increasing world population developing CVD a better understanding of the genes that predispose men to coronary heart disease and DCM is critical. Our findings show that the gene changes that contribute to an increased pro-inflammatory response in males are initiated within hours of infection in the spleen.

\section{Conclusions}

Men are at an increased risk of developing atherosclerosis, myocarditis, DCM and heart failure compared to women but the early immunological factors that drive the proinflammatory response remain unclear. In this study we found that the spleens of CVB3 infected male mice had a greater expression of genes associated with cholesterol and steroid influx and metabolism such as $\mathrm{PLA}_{2}, \mathrm{Msr} 1$ and squalene synthase compared to infected females. TSPO, the rate-limiting step for steroid synthesis and the AR were decreased in CVB3 infected males compared to infected females consistent with activation of the receptors. Thus, increased cholesterol metabolism in the spleen of males during the innate immune response to CVB3 infection may drive the testosterone-mediated proinflammatory response that we observe during acute CVB3 myocarditis, including mast cell and macrophage proliferation, $\mathrm{NF} \kappa \mathrm{B}$ activation and TLR4 signalling. These findings have important implications for other inflammatory CVDs that are increased in males.

\begin{abstract}
Abbreviations
AR: androgen receptor; CVB3: coxsackievirus; CVD: cardiovascular disease; DCM: myocarditis and dilated cardiomyopathy; FDR: false discover rate; HPRT: hypoxanthine phosphoribosyl transferase; ip: intraperitoneally; IPA: Ingenuity Pathway Analysis; LDL: Iow-density lipoprotein; LSD: lest significant difference; LV: left ventricular; PBS: phosphate buffered saline; pi: post infection; PFU: plaque forming units; PLA 2 : phospholipase $A_{2}$; q RT-PCR: quantitative real-time polymerase chain reaction; TLR: Toll-like receptor; RGF: relative gene expression; TSPO: translocator protein $18 \mathrm{kDa}$
\end{abstract}

\section{Acknowledgements}

The authors thank J Augusto Frisancho and Jaewook Shin for their technical assistance with the studies. This work was supported by National Institutes of Health Grants HL087033 (DF) and ES007062 (TRG).

\section{Author details}

'Department of Environmental Health Sciences, Johns Hopkins University Bloomberg School of Public Health, Baltimore, MD 21205, USA. ${ }^{2}$ Department of Pathology, Johns Hopkins University School of Medicine, Baltimore, MD 21205, USA. ${ }^{3}$ Department of Environmental Health Sciences, Mailman School of Public Health, Columbia University, New York, NY 10032, USA.

\section{Authors' contributions}

JAO and MJC contributed equally to this study, and are joint first authors. JAO carried out the microarray studies and analysis, participated in GRT-PCR assays and drafted the manuscript. MJC carried out the microarray studies and analysis and the qRT-PCR assays and analysis and helped draft the manuscript. AEG participated in the microarray studies and analysis and in
qRT-PCR analysis and conducted plaque assays. JBK conducted plaque assays. AB conducted ELISAs. DB conducted echocardiography and interpreted the results. KLG interpreted the echocardiography results and helped to draft the manuscript. TRG participated in the design of the study and the interpretation of data. DF conceived the study, participated in its design and coordination and helped to draft the manuscript. All authors read and approved the final manuscript.

\section{Competing interests}

The authors declare that they have no competing interests.

Received: 13 August 2010 Accepted: 21 February 2011

Published: 21 February 2011

\section{References}

1. WRITING GROUP MEMBERS, Lloyd-Jones D, Adams RJ, Brown TM, Carnethon M, Dai S, De Simone G, Ferguson TB, Ford E, Furie K, Gillespie C, American Heart Association Statistics Committee and Stroke Statistics Subcommittee, et al: Heart disease and stroke statistics- 2010 update: a report from the American Heart Association. Circulation 2010, 121 e46-e215, [Erratum in Circulation. 2010, 121: e260].

2. Wexler RK, Elton T, Pleister A, Feldman D: Cardiomyopathy: an overview. Am Fam Physician 2009, 79:778-784

3. Dec GW: Introduction to clinical myocarditis. In Myocarditis: From Bench to Bedside. Edited by: Cooper LT Jr, Totowa N. NY: Humana Press; 2003:257-281.

4. Regitz-Zagrosek V, Oertelt-Prigione S, Seeland U, Hetzer R: Sex and gender differences in myocardial hypertrophy and heart failure. Circ J 2010, 74:1265-1273.

5. Fairweather D, Frisancho-Kiss S, Rose NR: Sex differences in autoimmune disease from a pathologic perspective. Am J Pathol 2008, 173:600-609.

6. Cooper LT Jr: Myocarditis. N Engl J Med 2009, 360:1526-1538.

7. Cihakova D, Rose NR: Pathogenesis of myocarditis and dilated cardiomyopathy. Adv Immunol 2008, 99:95-114.

8. Schoub BD, Johnson S, McAnerney JM, Dos Santos IL, Klaassen KL: Epidemic Coxsackie B virus infection in Johannesburg, South Africa. $J$ Hyg (Lond) 1985, 95:447-455.

9. Dechkum N, Pangsawan Y, Jayavasu C, Saguanwongse S: Coxsackie B virus infection and myopericarditis in Thailand, 1987-1989. Southeast Asian J Trop Med Public Health 1998, 29:273-276.

10. Khetsuriani N, LaMonte-Fowlkes A, Oberste MS, Pallansch MA: Enterovirus surveillance- United States, 1970-2005. MMWR Surveill Summ 2006, 55:1-20.

11. Fairweather D, Rose NR: Coxsackievirus-induced myocarditis in mice: model of autoimmune disease for studying immunotoxicity. Methods 2007, 41:118-122.

12. Frisancho-Kiss S, Nyland JF, Davis SE, Frisancho JA, Barrett MA, Rose NR, Fairweather D: Sex differences in coxsackievirus B3-induced myocarditis: $\mathrm{IL}-12 \mathrm{R} \beta 1$ signaling and IFN- $\beta$ increase inflammation in males independent from STAT4. Brain Res 2006, 1126:139-147.

13. Frisancho-Kiss S, Davis SE, Nyland JF, Frisancho JA, Cihakova D, Rose NR, Fairweather D: Cutting Edge: Cross-regulation by TLR4 and T cell Ig mucin-3 determines sex differences in inflammatory heart disease. $J$ Immunol 2007, 178:6710-6714.

14. Frisancho-Kiss S, Coronado MJ, Frisancho JA, Lau VM, Rose NR, Klein SL, Fairweather D: Gonadectomy of male BALB/C mice increases Tim- $3^{+}$ alternatively activated M2 macrophages, Tim $-3^{+}$T cells, Th2 cells and Treg in the heart during acute coxsackievirus-induced myocarditis. Brain Behav Immun 2009, 23:649-657.

15. Fairweather D, Yusung S, Frisancho-Kiss S, Barrett M, Gatewood S, Steele R, Rose NR: IL-12R $\beta 1$ and TLR4 increase IL-1 $\beta$ and IL-18-associated myocarditis and coxsackievirus replication. J Immunol 2003, 170:4731-4737

16. Frisancho-Kiss S, Nyland JF, Davis SE, Barrett MA, Gatewood SJL, Njoku DB, Cihakova D, Silbergeld EK, Rose NR, Fairweather D: Cutting Edge: T cell Ig mucin-3 reduces inflammatory heart disease by increasing CTLA-4 during innate immunity. J Immunol 2006, 176:6411-6415.

17. Cihakova D, Barin JG, Afanasyeva M, Kimura M, Fairweather D, Berg M, Talor MV, Baldeviano GC, Frisancho-Kiss S, Gabrielson K, et al: Interleukin-13 protects against experimental autoimmune myocarditis by regulating macrophage differentiation. Am J Pathol 2008, 172:1195-1208. 
18. Page KR, Jedlicka AE, Fakheri B, Noland GS, Kesavan AK, Scott AL, Kumar N, Manabe YC: Myocabacterium-induced potentiation of type I immune responses and protection against malaria are host specific. Infect Immun 2005, 73:8369-8380.

19. Irizarry RA, Warren D, Spencer F, Kim IF, Biswal S, Frank BC, Gabrielson E, Garcia JGN, Geoghegan J, Germino G, et al: Multiple-laboratory comparison of microarray platforms. Nat Methods 2005, 2:345-350.

20. Rangasamy T, Misra V, Zhen L, Tankersley GC, Tuder RM, Biswal S: Cigarette smoke-induced emphysema in A/J mice associated with pulmonary oxidative stress, apoptosis of lung cells and global alterations in gene expression. Am J Physiol Lung Cell Mol Physiol 2009, 296:L888-L900.

21. Kass DA, Kitzman DW, Alvarez GE: The restoration of chronotropic competence in heart failure patients with normal ejection fraction (RESET) study: rationale and design. J Cardiac Fail 2010, 16:17-24.

22. Fairweather D, Frisancho-Kiss S, Gatewood S, Njoku D, Steele R, Barrett M, Rose NR: Mast cells and innate cytokines are associated with susceptibility to autoimmune heart disease following coxsackievirus B3 infection. Autoimmunity 2004, 37:131-145.

23. Isensee J, Witt H, Pregla R, Hetzer R, Regitz-Zagrosek V, Noppinger PR: Sexually dimorphic gene expression in the heart of mice and men. $J \mathrm{Mol}$ Med 2008, 86:61-74.

24. Waxman DJ, Holloway MG: Sex differences in the expression of hepatic drug metabolizing enzymes. Mol Pharmacol 2009, 76:215-228.

25. Cederbaum A: Nrf2 and antioxidant defense against CYP2E1 toxicity. Expert Opin Drug Metab Toxicol 2009, 5:1223-1244.

26. Alvarez BV, Johnson DE, Sowah D, Soliman D, Light PE, Xia Y, Karmazyn M Casey JR: Carbonic anhydrase inhibition prevents and reverts cardiomyocyte hypertrophy. J Physiol 2007, 579:127-145.

27. Quaye IK: Haptoglobin, inflammation and disease. Trans $R$ Soc Trop Med Hyg 2008, 102:735-742.

28. Thum T, Borlak J: Testosterone, cytochrome P450, and cardiac hypertrophy. FASEB J 2002, 16:1537-1549.

29. Yeap BB, Krueger RG, Leedman PJ: Differential posttranscriptional regulation of androgen receptor gene expression by androgen in prostate and breast cancer cells. Endocrinology 1999, 140:3282-3291.

30. Veenman L, Gavish M: The peripheral-type benzodiazepine receptor and the cardiovascular system. Implications for drug development. Pharmacol Ther 2006, 110:503-524.

31. Packard CJ: Lipoprotein-associated phospholipase $A_{2}$ as a biomarker of coronary heart disease and a therapeutic target. Curr Opin Cardiol 2009, 24:358-363.

32. Do R, Kiss RS, Gaudet D, Engert JC: Squalene synthase: a critical enzyme in the cholesterol biosynthesis pathway. Clin Genet 2009, 75:19-29.

33. Liao P, Georgakopoulos D, Kovacs A, Zheng M, Lerner D, Pu H, Saffitz J, Chien K, Xiao R-P, Kass DA, Wang Y: The in vivo role of p38 MAP kinases in cardiac remodeling and restrictive cardiomyopathy. Proc Nat Acad Sci USA 2001, 98:12283-12288.

34. Boheler KR, Volkova M, Morrell C, Garg R, Zhu Y, Margulies K, Seymour A-M, Lakatta EG: Sex- and age-dependent human transcriptome variability: Implications for chronic heart failure. Proc Nat Acad Sci USA 2003, 100:2754-2759.

35. Karin $\mathrm{N}$ : The multiple faces of $\mathrm{CXCL12}$ (SDF-1 $\beta$ ) in the regulation of immunity during health and disease. J Leukoc Biol 2010, 88:463-473.

36. Fairweather D, Frisancho-Kiss S, Yusung SA, Barrett MA, Gatewood SJL, Davis SE, Njoku DB, Rose NR: IFN- $\beta$ protects against chronic viral myocarditis by reducing mast cell degranulation, fibrosis, and the profibrotic cytokines TGF- $\beta_{1}, I L-1 \beta$ and IL-4 in the heart. Am J Pathol 2004, 165:1883-1894.

37. Diedrich M, Tadic J, Mao L, Wacker MA, Nebrich G, Hetzer R, RegitzZagrosek V, Klose J: Heart protein expression related to age and sex in mice and humans. Int J Mol Med 2007, 20:865-874.

38. Zordoky BN, El-Kadi AO: Modulation of cardiac and hepatic cytochrome P450 enzymes during heart failure. Curr Drug Metab 2008, 9:122-128.

39. Tabet F, Rye K-A: High-density lipoproteins, inflammation and oxidative stress. Clin Sci 2009, 116:87-98.

40. Burke JE, Dennis EA: Phospholipase $A_{2}$ structure/function, mechanism, and signaling. J Lipid Res 2009, 50:S237-S242.

41. White $\mathrm{H}$ : Why inhibition of lipoprotein-associated phospholipase $A_{2}$ has the potential to improve patient outcomes. Curr Opin Cardiol 2010, 25:299-301.
42. Levy $R$ : The role of cytosolic phospholipase $A_{2}$-alpha in regulation of phagocytic functions. Biochim Biophys Acta 2006, 1761:1323-1334.

43. Biolard E, Lai Y, Larabee K, Balestrieri B, Ghomashchi F, Fujioka D, Gobezie R, Coblyn JS, Weinblatt ME, Massarotti EM, et al: A novel anti-inflammatory role for secretory phospholipase $A_{2}$ in immune complex-mediated arthritis. EMBO Mol Med 2010, 2:172-187.

44. Mounier CM, Wendum D, Greenspan E, Flejou J-F, Rosenberg DW, Lambeau G: Distinct expression pattern of the full set of secreted phospholipases $A_{2}$ in human colorectal adenocarcinomas: SPLA $A_{2}-111$ as a biomarker candidate. Br J Cancer 2008, 98:587-595.

45. Henderson RJ, Wasan KM, Leon CG: Haptoglobin inhibits phospholipid transfer protein activity in hyperlipidemic human plasma. Lip Health Dis 2009, 8:27.

46. Von Haehling S, Schefold JC, Lainscak M, Doehner W, Anker SD: Inflammatory biomarkers in heart failure revisited: much more than innocent bystanders. Heart Failure Clin 2009, 5:549-560.

\section{doi:10.1186/2042-6410-2-2}

Cite this article as: Onyimba et al:: The innate immune response to coxsackievirus B3 predicts progression to cardiovascular disease and heart failure in male mice. Biology of Sex Differences 2011 2:2.

\section{Submit your next manuscript to BioMed Central and take full advantage of:}

- Convenient online submission

- Thorough peer review

- No space constraints or color figure charges

- Immediate publication on acceptance

- Inclusion in PubMed, CAS, Scopus and Google Scholar

- Research which is freely available for redistribution

Submit your manuscript at www.biomedcentral.com/submit
C) Biomed Central 